\title{
Universal physics of three bosons with isospin
}

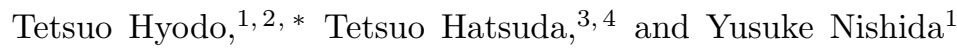 \\ ${ }^{1}$ Department of Physics, Tokyo Institute of Technology, Ookayama, Meguro, Tokyo 152-8551, Japan \\ ${ }^{2}$ Yukawa Institute for Theoretical Physics, Kyoto University, Kyoto 606-8502, Japan \\ ${ }^{3}$ Theoretical Research Division, Nishina Center, RIKEN, Wako, Saitama 351-0198, Japan \\ ${ }^{4}$ Kavli IPMU (WPI), University of Tokyo, Chiba 277-8583, Japan
}

(Dated: August 6, 2018)

\begin{abstract}
We show that there exist two types of universal phenomena for three-boson systems with isospin degrees of freedom. In the isospin symmetric limit, there is only one universal three-boson bound state with the total isospin one, whose binding energy is proportional to that of the two-boson bound state. With large isospin symmetry breaking, the standard Efimov states of three identical bosons appear at low energies. Both phenomena can be realized by three pions with the pion mass appropriately tuned in lattice QCD simulations, or by spin-one bosons in cold atom experiments. Implication to the in-medium softening of multi-pion states is also discussed.

PACS numbers: 03.65.Ge, 11.30.Rd, 21.65.Jk, 67.85.Fg
\end{abstract}

Introduction. The properties of particles interacting with a large scattering length are universal, i.e., they are determined irrespective of the short range behavior of the interaction. In particular, three-particle systems with a large two-body scattering length lead to the emergence of the Efimov states [1], which have been extensively studied in cold atom physics [2]. Moreover, in condensed matter physics, collective excitations in quantum magnets are shown to exhibit the Efimov effect [3].

Since the intrinsic energy scale of the system is not relevant for such universal phenomena, they could be also realized in strong interaction governed by quantum chromodynamics (QCD) as far as suitable conditions are met. In fact, a theoretical possibility of achieving the Efimov effect in the three-nucleon system by making a slight modification of the light quark masses was discussed in the framework of the effective field theory of QCD [4]. The existence of $X(3872)$ near the $D^{0} \bar{D}^{* 0}$ $\bar{D}^{0} D^{* 0}$ threshold has motivated a search for the universal three-boson bound state, $D^{0} D^{0} \bar{D}^{* 0}$. However, the coupled channel effect reduces the attraction and hence no universal bound state was found in such a system [5].

In this Rapid Communication, we show that two universal phenomena arise for three bosons with three internal degrees of freedom (such as the pion $\pi$ ) if the twobody scattering length is large. In the real world, the $s$ wave $\pi \pi$ scattering lengths are as small as $a_{I=0} \sim-0.31$ $\mathrm{fm}$ and $a_{I=2} \sim 0.06 \mathrm{fm}[\underline{6}]$ due to the Nambu-Goldstone nature of the pion, so that the condition of the universality does not hold. Indeed, the $I=0 s$-wave $\pi \pi$ scattering in the real world leads to the $\sigma$ resonance far above the threshold [7]. However, if the quark mass is increased artificially, the situation changes: the lattice QCD simulations [8] and chiral effective theory [9] show that the $\sigma$ meson becomes a bound state for heavy quark masses. This implies that there is an intermediate region of the

\footnotetext{
* hyodo@yukawa.kyoto-u.ac.jp
}

quark mass where the $\sigma$ meson lies close to the $\pi \pi$ threshold and the $\pi \pi$ interaction is characterized by a large scattering length. In such a region, the three-pion system exhibits universal phenomena, which can be tested by simulating the three pions on the lattice by changing the quark mass. From the point of view of the statistical noise, three pions with heavy quark mass are much less costly than the three nucleons with light quark mass [10]. In this sense, the three-pion system is an ideal testing ground for the universal physics in QCD.

Universal physics with the isospin symmetry. Let us first consider the three-pion system with exact isospin symmetry. We assume that, by an appropriate tuning of the quark mass, only the $s$-wave $\pi \pi$ scattering length in the $I=0$ channel, $\left|a_{I=0}\right|$, becomes much larger than the typical length scale $R$ characterized by the interaction range. In addition, we consider momentum $p$ sufficiently smaller than $1 / R$, so that the pions can be treated as non-relativistic particles with a contact interaction. Then, the system is represented by the universal zerorange theory [11] with iso-scalar interaction:

$$
\begin{aligned}
\mathcal{L} & =\sum_{i=1,2,3} \phi_{i}^{\dagger}\left(i \partial_{t}+\frac{\nabla^{2}}{2 m}\right) \phi_{i}+v\left|\sum_{i=1,2,3} \phi_{i} \phi_{i}\right|^{2} \\
& =\sum_{j=0, \pm} \pi_{j}^{\dagger}\left(i \partial_{t}+\frac{\nabla^{2}}{2 m}\right) \pi_{j}+v\left|\pi_{0} \pi_{0}+2 \pi_{-} \pi_{+}\right|^{2},
\end{aligned}
$$

where $\pi_{ \pm}=\left(\phi_{1} \mp i \phi_{2}\right) / \sqrt{2}$ and $\pi_{0}=\phi_{3}$. Here $m$ and $v$ are the pion mass and the bare coupling constant, respectively. The constant interaction is a consequence of the explicit symmetry breaking by the nonzero quark mass [12]. It dominates over the momentum-dependent interaction near the $\pi \pi$ threshold. After the renormalization, the two-pion scattering $t$-matrix in $I=0$ is characterized only by the scattering length $a=a_{I=0}$ as

$$
i t_{0}(p)=\frac{8 \pi}{m} \frac{i}{\frac{1}{a}-\sqrt{\frac{p^{2}}{4}-m p_{0}-i 0^{+}}},
$$


while the $I=2$ component vanishes identically, $i t_{2}(p)=$ 0 .

Now we consider the three-pion system. By combining one pion with the $\pi \pi$ pair in $I=0$, the whole system has $I=1$, which can also be constructed by the $\pi \pi$ pair in $I=2$. Thus, the three-pion system in the isospin symmetric limit is a coupled-channel problem. Different pairings of two pions are related with each other through the recoupling coefficients as

$$
\begin{gathered}
\left(\begin{array}{c}
\left|\pi \otimes[\pi \otimes \pi]_{I=0}\right\rangle_{I=1} \\
\left|\pi \otimes[\pi \otimes \pi]_{I=2}\right\rangle_{I=1}
\end{array}\right)=\Omega\left(\begin{array}{l}
\left|[\pi \otimes \pi]_{I=0} \otimes \pi\right\rangle_{I=1} \\
\left|[\pi \otimes \pi]_{I=2} \otimes \pi\right\rangle_{I=1}
\end{array}\right), \\
\Omega=\left(\begin{array}{cc}
1 / 3 & \sqrt{5} / 3 \\
\sqrt{5} / 3 & 1 / 6
\end{array}\right) .
\end{gathered}
$$

Then, the coupled-channel three-body scattering amplitude with total energy $E$ obeys the integral equation

$$
\begin{aligned}
& i T_{I J}(E ; k, p) \\
& =i \Omega_{I J} G(P-k-p) \\
& \quad-\sum_{K=0,2} \int_{q} T_{I K}(E ; k, q) t_{K}(P-q) \Omega_{K J} G(q) G(P-q-p),
\end{aligned}
$$

where $P=(E, \mathbf{0}), k$, and $p$ are the four momenta of the total system, of the initial spectator pion, and of the final spectator pion, respectively. The pion propagator with momentum $p$ is written as $G(p)=1 /\left(p_{0}-\varepsilon_{\boldsymbol{p}}+i 0^{+}\right)$with $\varepsilon_{\boldsymbol{p}}=\boldsymbol{p}^{2} / 2 m$.

Because the $I=2$ two-body amplitude vanishes in the present case, the coupled-channel three-body equation reduces to a single-channel equation. For the on-shell $T$ matrix $T^{\mathrm{on}}(E ; \boldsymbol{k}, \boldsymbol{p})=\left.T_{00}(E ; k, p)\right|_{k_{0}=\varepsilon_{\boldsymbol{k}}, p_{0}=\varepsilon_{\boldsymbol{p}}}$, we have

$$
\begin{aligned}
& T^{\mathrm{on}}(E ; \boldsymbol{k}, \boldsymbol{p}) \\
& =\frac{1}{3} g(E, \boldsymbol{k}, \boldsymbol{p})+\frac{1}{3} \int_{\boldsymbol{q}} T^{\mathrm{on}}(E ; \boldsymbol{k}, \boldsymbol{q}) \\
& \quad \times g(E, \boldsymbol{q}, \boldsymbol{p}) \frac{8 \pi}{m} \frac{1}{\frac{1}{a}-\sqrt{\frac{\boldsymbol{q}^{2}}{4}-m\left(E-\varepsilon_{\boldsymbol{q}}\right)-i 0^{+}}},
\end{aligned}
$$

where $g(E, \boldsymbol{k}, \boldsymbol{p})=\left.G(P-k-p)\right|_{k_{0}=\varepsilon_{\boldsymbol{k}}, p_{0}=\varepsilon_{\boldsymbol{p}}}$. Because of the existence of the $I=2$ component, the probability of finding an $I=0$ pair after the particle exchange is reduced from unity to $1 / 3$ in the right hand side. This is a major difference between identical bosons and the bosons with internal degrees of freedom.

Three-pion bound states can be investigated by solving the homogeneous equation. After the $s$-wave projection, the scattering amplitude depends only on the magnitude of the spectator momenta. At the energy of the bound state pole $E=-B_{3}$ with $B_{3}>0$, the amplitude factorizes as $T^{\mathrm{on}}(E ;|\boldsymbol{k}|,|\boldsymbol{p}|)=z^{*}(|\boldsymbol{k}|) z(|\boldsymbol{p}|) /\left(E+B_{3}\right)$, and the eigenvalue equation for $z(|\boldsymbol{p}|)$ leads to

$$
\begin{aligned}
z(|\boldsymbol{p}|)= & \frac{2}{\lambda \pi} \int_{0}^{\infty} d|\boldsymbol{q}| \frac{|\boldsymbol{q}|}{|\boldsymbol{p}|} \ln \left(\frac{\boldsymbol{q}^{2}+\boldsymbol{p}^{2}+|\boldsymbol{q}||\boldsymbol{p}|+m B_{3}}{\boldsymbol{q}^{2}+\boldsymbol{p}^{2}-|\boldsymbol{q}||\boldsymbol{p}|+m B_{3}}\right) \\
& \times \frac{z(|\boldsymbol{q}|)}{\sqrt{\frac{3}{4} \boldsymbol{q}^{2}+m B_{3}}-\frac{1}{a}}
\end{aligned}
$$

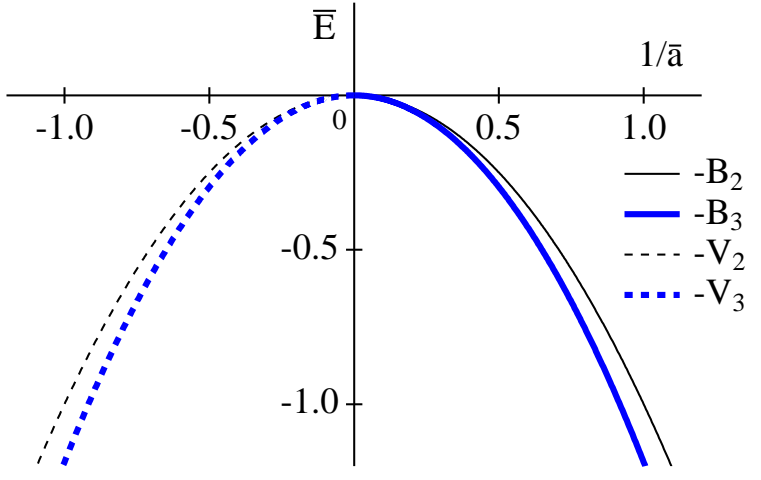

FIG. 1. (Color online) Energies of the bound and virtual states in the isospin symmetric limit with $\bar{E}=\operatorname{sgn}(E)|m E|^{4}$ and $\bar{a}=\operatorname{sgn}(a)|a|^{4}$ in arbitrary units. The thick (thin) solid line represents the energy of the three-pion (two-pion) bound state, and the thick (thin) dashed line the energy of the threepion (two-pion) virtual state.

where we introduce a factor $\lambda$ for later use; it is $\lambda=3$ in the present case. In the two-pion system, there is a bound state at $E=-1 /\left(m a^{2}\right) \equiv-B_{2}$ for a positive inverse scattering length $1 / a$, as seen in Eq. (2). This means that the lowest threshold in the three-pion channel is $E=0$ (three-body breakup) for negative $1 / a$ and $E=-B_{2}$ (two-body decay into $\pi$ and a $\pi \pi$ bound state) for positive $1 / a$. Solving Eq. (44) numerically, we find one bound state for the three pions at

$$
B_{3}=\frac{1.04391}{m a^{2}} \text { for } 1 / a>0 .
$$

On the other hand, no bound state is generated for $1 / a<$ 0 . Because there is no scale other than the scattering length, it is a universal result that there is a three-pion bound state whose binding energy is proportional to that of the two-pion system. The results are illustrated in Fig. 1]

We find that both the three-pion and two-pion bound states vanish at $1 / a=0$. We can investigate the fate of the bound state pole in the negative $1 / a$ region. It is known that the two-body bound state pole turns into a virtual state, which appears in the lower half-plane of the complex momentum space, namely, the second Riemann sheet of the complex energy plane [13]. The first (second) Riemann sheet can be reached by a rotation of the binding energy $B_{2} \rightarrow B_{2} e^{i \theta}$ with $-\pi \leq \theta<\pi(\pi \leq \theta \leq 2 \pi$, $-2 \pi<\theta<-\pi)$. In fact, when a two-body bound state solution is obtained for a given $1 / a>0$ at $E=-B_{2}$, there is also a solution for the corresponding $-1 / a<0$ at $E=-B_{2} e^{2 \pi i}=-V_{2}$. Thus, we have a two-pion virtual state for a negative $1 / a$ at $V_{2}=1 /\left(m a^{2}\right)$.

We can also search for three-body virtual state solutions by rotating the binding energy as $B_{3} \rightarrow B_{3} e^{i \theta}$. In general, the distribution of the singularities of the integrand of Eq. (4) depends on the angle $\theta$, so that 
the integration contour should be taken carefully. In the present case, however, we can rewrite the equation by using the momentum variables $|\tilde{\boldsymbol{q}}|=|\boldsymbol{q}| e^{-i \theta / 2}$ and $z\left(|\tilde{\boldsymbol{q}}| e^{i \theta / 2}\right)=\tilde{z}(|\tilde{\boldsymbol{q}}|)$. Noting that the integrand vanishes at $|\tilde{\boldsymbol{q}}| \rightarrow \infty$, we obtain the same form as Eq. (4) with the replacement $1 / a \rightarrow e^{-i \theta / 2} / a$. Namely, the rotation of the energy by $e^{i \theta}$ is equivalent to rotating the inverse scattering length by $e^{-i \theta / 2}$. Thus, as in the case of the two-body system, a solution for the virtual state $(\theta=2 \pi)$ is obtained by the bound state solution with the opposite sign of the scattering length; $V_{3}=1.04391 /\left(m a^{2}\right)$ for $1 / a<0$.

We have numerically checked that there is no resonance state in the second Riemann sheet for both positive and negative scattering lengths. In fact, the existence of a resonance pole at a certain $\theta$ indicates the existence of a pole at $\theta+2 \pi$ with the opposite sign of the scattering length. However, if $B_{3} e^{i \theta}$ is in the second Riemann sheet, $B_{3} e^{i(\theta+2 \pi)}$ is in the first Riemann sheet, where the existence of poles (except for bound states) is forbidden by causality [13]. Thus, the present system does not allow resonance solutions.

Universal physics with the isospin breaking. We now consider the effect of the isospin symmetry breaking. In the real world, the charged $\pi_{ \pm}$states are heavier than the neutral $\pi_{0}$ mainly due to the electromagnetic interaction. We assume the same ordering even for the heavy quark mass and focus on the energy window where the effect of $\pi_{ \pm}$can be negligible. Then the system reduces to a single-channel problem of three neutral pions. This is nothing but the case where the Efimov effect arises [2].

Let us briefly show how it occurs. Because of the absence of the channel coupling, we remove the $1 / 3$ factor in the right hand side of Eq. (3) and the corresponding eigenvalue equation is Eq. (4) with $\lambda=1$ as

$$
\begin{aligned}
z(|\boldsymbol{p}|)= & \frac{2}{\pi} \int_{0}^{\infty} d|\boldsymbol{q}| \frac{|\boldsymbol{q}|}{|\boldsymbol{p}|} \ln \left(\frac{\boldsymbol{q}^{2}+\boldsymbol{p}^{2}+|\boldsymbol{q}||\boldsymbol{p}|+m B_{3}}{\boldsymbol{q}^{2}+\boldsymbol{p}^{2}-|\boldsymbol{q}||\boldsymbol{p}|+m B_{3}}\right) \\
& \times \frac{z(|\boldsymbol{q}|)}{\sqrt{\frac{3}{4} \boldsymbol{q}^{2}+m B_{3}}-\frac{1}{a}} f_{\Lambda}(|\boldsymbol{q}|),
\end{aligned}
$$

where $a=a_{\pi_{0} \pi_{0}}$ and $m$ is the mass of $\pi_{0}$. Here we introduce the cutoff function $f_{\Lambda}(|\boldsymbol{q}|)$ with $f_{\Lambda} \rightarrow 0$ for $|\boldsymbol{q}| \gg \Lambda$, so that the equation is well defined. For momentum much lower than $\Lambda$, the system exhibits the universal physics with infinitely many bound states at $1 / a=0$. The spectrum in this region is independent of the cutoff, and is characterized by the Efimov parameter $\kappa_{*}$; the energy of the $n$th excited state is $E_{n} \rightarrow-e^{-2 \pi n / s_{0}} \kappa_{*}^{2} / m$ with $s_{0}=1.00624$ for a sufficiently large $n$. In Fig. 2, we show by the solid lines the energies of the three-pion bound states as functions of $1 / a$ with appropriate powers for visualization.

To study the trajectory of the bound state pole after the dissociation, we search for the resonance solutions in the second Riemann sheet as discussed in Refs. [14, 15]. For a resonance, the residue of the pole should be $z^{*}(|\boldsymbol{k}|) z(|\boldsymbol{p}|) \rightarrow z(|\boldsymbol{k}|) z(|\boldsymbol{p}|)$, but the eigenvalue

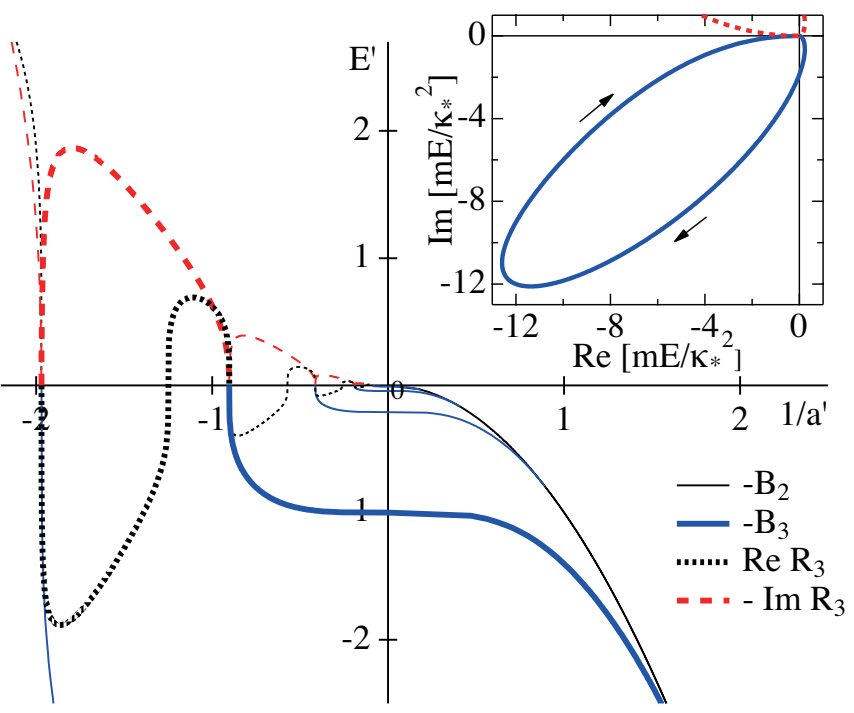

FIG. 2. (Color online) Energies of the bound states of the three $\pi_{0}$ system with the large isospin breaking. The solid lines represent the energies of the bound states, and the dotted (dashed) lines represent the real parts (imaginary parts with the opposite sign) of the resonance poles. Both the axes are normalized to be dimensionless by the Efimov parameter $\kappa_{*}$ as $E^{\prime}=\operatorname{sgn}(E)\left|m E / \kappa_{*}^{2}\right|^{1 / 4}$ and $a^{\prime}=\operatorname{sgn}(a)\left|a \kappa_{*}\right|^{1 / 4}$. The inset shows the trajectories of the pole positions in the complex energy plane for a period corresponding to $-0.663293 \geq$ $1 /\left(a \kappa_{*}\right) \geq-15.0530$. The solid (dashed) line stands for the resonance (anti-resonance) state. As the inverse scattering length is decreased, the pole moves to the direction indicated by the arrows.

equation (6) remains the same. In the present case, the rotation of the binding energy $B_{3} \rightarrow B_{3} e^{i \theta}$ is equivalent to rotating $1 / a \rightarrow e^{-i \theta / 2} / a$ and $\Lambda \rightarrow \Lambda e^{-i \theta / 2}$. In general, the cutoff function can have a pole. For $f_{\Lambda}(|\boldsymbol{q}|)=\Lambda /(|\boldsymbol{q}|+\Lambda)$, the pole position after the phase rotation is $|\tilde{\boldsymbol{q}}|=-\Lambda e^{-i \theta / 2}$ which does not cross the integration path for $-2 \pi<\theta<2 \pi$ (therefore, our analysis cannot confirm or exclude the existence of virtual states). With a different form of the function $f_{\Lambda}(|\boldsymbol{q}|)$, however, we need to treat the singularities properly, by adding the residue terms depending on the rotation angle $\theta$, in order to obtain the correct results.

By solving the eigenvalue equation, we find that an Efimov bound state pole turns into a resonance (and an anti-resonance expressed by the complex conjugate of the resonance pole) when the binding energy crosses zero for $1 / a<0$. The real and imaginary parts of the pole position are shown in Fig. 2. As we decrease 1/a, the pole first moves to the higher energy with increasing the width, but at some point it changes the direction and goes to the subthreshold region [15]. Interestingly, as we further decrease $1 / a$, the pole again moves toward the threshold with decreasing the imaginary part, and finally reaches the threshold when the next [(n-1)th] Efimov bound state comes to the threshold. This pole trajectory is shown in the inset of Fig. 2. In contrast, when the Efimov 
TABLE I. Classification of the universal three-body bound states with respect to the parameter $\lambda$ in Eq. (4).

\begin{tabular}{ccc}
\hline \hline$\lambda<2.41840$ & $2.41840<\lambda<3.66811$ & $3.66811<\lambda$ \\
\hline Efimov states & Single universal state & None \\
\hline \hline
\end{tabular}

bound state dissociates with $1 / a>0$, the three-body problem is effectively reduced to the two-body problem of a pion and a two-pion bound state. According to the universal two-body physics, the bound state turns into a virtual state, in accordance with Ref. [14]. In this respect, the direct transition from the bound state to the resonance for $1 / a<0$ is a peculiar feature in the three-body system. Moreover, the cyclic pole trajectory is in contrast to the usual two-body resonance which goes away from the real axis when the attraction is weakened. This behavior also shows that there is only one resonance at a given $1 / a<0$, although the number of the bound states is infinite at $1 / a=0$.

Classification of the universal physics. We have seen that the number of the universal bound states is only one for the isospin symmetric case and infinity for the isospin breaking case. The difference stems from the factor $\lambda$ in Eq. (4) which represents the effective reduction of the attraction by the coupled-channel effect. The solution of Eq. (4) is classified by the value of $\lambda$ in Table I. For $\lambda$ smaller than $4 \pi / 3 \sqrt{3} \approx 2.41840$, the Efimov effect occurs. The single universal bound state is obtained for $\lambda$ until 3.66811, above which no bound state appears. The $D^{0} D^{0} \bar{D}^{* 0}$ three-body system considered in Ref. [5] corresponds to $\lambda=4$ and hence there is no bound state. The three-pion system with heavy quark mass is a unique system which is capable of realizing both the Efimov states and the single universal state.

Interpolation by a model. The two types of universal state discussed above can be described under the modification of the zero-range theory in Eq. (1) by introducing a finite mass difference between $\pi_{ \pm}$and $\pi_{0}$, i.e., $\nabla^{2} /(2 m) \rightarrow \nabla^{2} /\left(2 m_{j}\right)-m_{j} c^{2}$ with $\Delta=\left(m_{ \pm}-m_{0}\right) c^{2}>$ 0 . The length scale associated with the mass difference is $1 / \sqrt{m_{0} \Delta}$. If this is much larger than the typical interaction range $R$, we should have the infinitely many Efimov bound states for $p \ll \sqrt{m_{0} \Delta}$, while only a single bound sate arises for $\sqrt{m_{0} \Delta} \ll p \ll 1 / R$. Measuring the energy from the $\pi_{0} \pi_{0}$ threshold, we obtain the two-body amplitude as

$$
\begin{aligned}
i t(p)= & \frac{8 \pi}{m_{0}} i\left\{\frac{1}{a}-\frac{1}{3} \sqrt{\frac{\boldsymbol{p}^{2}}{4}-m_{0} p_{0}-i 0^{+}}-\frac{2 m_{ \pm}}{3 m_{0}}\right. \\
& \left.\times\left[\sqrt{\frac{\boldsymbol{p}^{2}}{4}-m_{ \pm} p_{0}+2 m_{ \pm} \Delta-i 0^{+}}-\sqrt{2 m_{ \pm} \Delta}\right]\right\}^{-1} .
\end{aligned}
$$

For $\Delta / E \rightarrow 0$, we recover Eq. (2) in the isospin symmetric limit if the mass ratio $m_{ \pm} / m_{0}$ is close to unity.

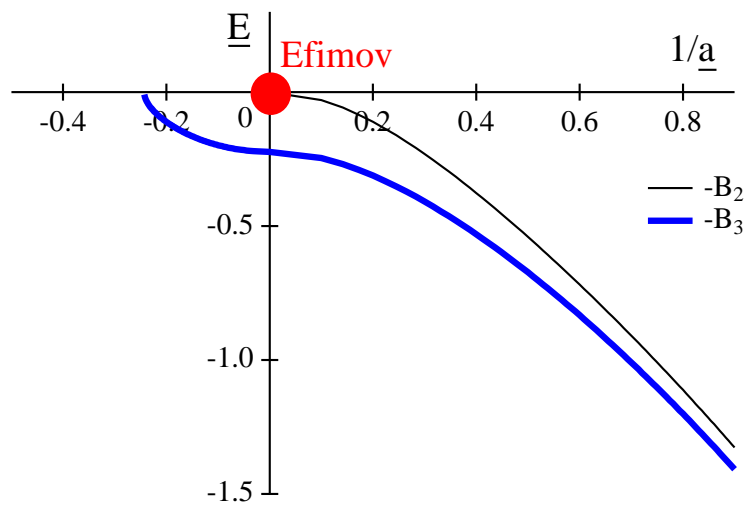

FIG. 3. (Color online) Binding energies from the interpolation model with the mass ratio $m_{ \pm} / m_{0}=1.03403$. The filled circle at origin expresses the accumulation of the Efimov bound states. The axes are given by the dimensionless quantities $\underline{E}=\operatorname{sgn}(E)|E / \Delta|^{1 / 2}$ and $1 / \underline{a}=\operatorname{sgn}(a)\left|1 / a \sqrt{m_{0} \Delta}\right|^{1 / 2}$.

For $\Delta / E \rightarrow \infty$, we obtain the $\pi_{0} \pi_{0}$ amplitude with $1 / a \rightarrow 3 / a$, and also with an effective range $r_{e}=$ $-2\left(m_{ \pm} / m_{0}\right)^{2} / \sqrt{2 m_{ \pm} \Delta}$. Therefore, this model interpolates two universal physics with the variation of the energy scale $E$. In addition, a cutoff scale for the Efimov physics at $\Delta / E \rightarrow \infty$ is naturally introduced by the mass difference in the form of $r_{e}$. Note that the behavior outside the universal region should be determined by the underlying dynamics, and this model provides one of the possible scenarios.

In Fig. 3, we show the spectrum of the above interpolation model with the mass ratio being $m_{ \pm} / m_{0}=$ 1.03403. The infinitely many Efimov states appear in the vicinity of the origin where the isospin breaking scale is much larger than the eigenenergies. For the $1 / a \gg \sqrt{m_{0} \Delta}$ region, there is only one bound state at $B_{3}=1.02982 /\left(m_{0} a^{2}\right)$ which is slightly different from Eq. (5) because of the mass ratio $m_{ \pm} / m_{0} \neq 1$. In the present model, we find that the lowest level of the Efimov states is continuously connected to the single universal bound state in the large $1 /$ a region.

Discussions. As mentioned in the Introduction, the three-pion system with heavy quark mass can be simulated in lattice QCD. First, we need to tune the quark mass so that the two pions in the $s$-wave and $I=0$ channel form a shallow bound state which we call $\sigma$. Then, we search for the bound state in the three-pion system in the $I=1$ channel which we call $\pi^{*}$. For the isospin symmetric case, the ratio of the binding energies between $\pi^{*}$ and $\sigma$ will be precisely $B_{3} / B_{2}=1.04391$ in the universal region. The confirmation of the Efimov states which appear under the isospin symmetry breaking would be a numerical challenge because the binding energies are separated by a large factor, 515 .

The universal three-pion bound states are unlikely to be realized in the vacuum because of the small $\pi \pi$ scattering length for light quark masses. On the other hand, 
in the nuclear medium, one can expect an effective increase of the $\pi \pi$ attraction due to partial restoration of chiral symmetry [16], so that the $\sigma$ resonance approaches and reaches the $\pi \pi$ threshold before the entire symmetry restoration taking place [17, 18]. Around the baryon density where $\sigma$ and $\pi \pi$ are almost degenerate, the universal three-pion state $\pi^{*}$ would simultaneously appear. Experimental confirmation of such novel bound states $(\sigma$ and $\pi^{*}$ ) in the nuclear medium is an interesting problem to be explored in hadron-nucleus and nucleus-nucleus reactions.
Finally, we note that our results have direct relevance to cold atom physics. In such a case, one can consider a spin-one bosonic atom instead of the isospin-one pion. Because the interaction between cold atoms can in principle be controlled, it is also interesting to study universal three-boson physics with spin degrees of freedom in cold atom experiments.

The authors thank Yoichi Ikeda for helpful discussions. This work was partially supported by JSPS KAKENHI Grants No. 24105702, No. 24740152, and No. 25887020, by the Yukawa International Program for Quark-Hadron Sciences (YIPQS), and by RIKEN iTHES Project.
[1] V. Efimov, Phys. Lett. B 33, 563 (1970).

[2] E. Braaten and H.-W. Hammer, Phys. Rept. 428, 259 (2006); Ann. Phys. (NY) 322, 120 (2007).

[3] Y. Nishida, Y. Kato, and C. D. Batista, Nat. Phys. 9, 93 (2013).

[4] E. Braaten and H.-W. Hammer, Phys. Rev. Lett. 91, 102002 (2003).

[5] E. Braaten and M. Kusunoki, Phys. Rev. D 69, 074005 (2004).

[6] R. García-Martín, R. Kamiński, J. Peláez, J. Ruiz de Elvira, and F. Ynduráin, Phys. Rev. D 83, 074004 (2011).

[7] J. Beringer et al. (Particle Data Group), Phys. Rev. D 86, 010001 (2012).

[8] T. Kunihiro et al. (SCALAR Collaboration), Phys. Rev. D 70, 034504 (2004).

[9] C. Hanhart, J. R. Pelaez, and G. Rios, Phys. Rev. Lett. 100, 152001 (2008).
[10] G. P. Lepage, in Proceedings of the 1989 Theoretical Advanced Study Institute (TASI), CLNS-89-971 (unpublished).

[11] E. Braaten, M. Kusunoki, and D. Zhang, Ann. Phys. (NY) 323, 1770 (2008).

[12] S. Scherer and M. R. Schindler, Lect. Notes Phys. 830, 1 (2012).

[13] J. R. Taylor, Scattering Theory: The Quantum Theory on Nonrelativistic Collisions (Wiley, New York, 1972).

[14] M. T. Yamashita, T. Frederico, A. Delfino, and L. Tomio, Phys. Rev. A 66, 052702 (2002).

[15] F. Bringas, M. T. Yamashita, and T. Frederico, Phys. Rev. A 69, 040702 (2004).

[16] R. S. Hayano and T. Hatsuda, Rev. Mod. Phys. 82, 2949 (2010).

[17] T. Hatsuda, T. Kunihiro, and H. Shimizu, Phys. Rev. Lett. 82, 2840 (1999).

[18] T. Hyodo, D. Jido, and T. Kunihiro, Nucl. Phys. A848, 341 (2010). 